\title{
Single nucleotide polymorphisms in glutathione S-transferase P1 and $M 1$ genes and overall survival of patients with ovarian serous cystadenocarcinoma treated with chemotherapy
}

\author{
LAN-XIANG CONG ${ }^{1,2}$, XIANG-HONG ZHAI ${ }^{3}$, FENG-XIA WU ${ }^{4}$, DONG-YI ZHU ${ }^{1,2}$ and AN-CONG WANG ${ }^{1,2}$ \\ Departments of ${ }^{1}$ Reproductive Medicine and ${ }^{2}$ Obstetrics and Gynecology, Linyi People's Hospital, Linyi, Shandong 276003; \\ ${ }^{3}$ Department of Obstetrics and Gynecology, Linyi Health School, Linyi, Shandong 276000; \\ ${ }^{4}$ Department of Anatomy, Shandong University, Jinan, Shandong 250012, P.R. China
}

Received November 25, 2014; Accepted January 4, 2016

DOI: $10.3892 / 01.2016 .4223$

\begin{abstract}
The effects of platinum-based drugs are controlled by genes that are involved in DNA detoxification, including glutathione S-transferase (GST)P1 and GSTM1, which have been associated with increased benefits in the chemotherapeutic treatment of patients with ovarian cancer. The present study assessed the effect of single nucleotide polymorphisms in GST genes on the overall survival (OS) of patients with ovarian serous cystadenocarcinoma that were treated with chemotherapy. A total of 95 patients received treatment with a carboplatin-based or alternative chemotherapy. Polymorphisms in the patients were genotyped using the following methods: Pyrosequencing, to identify GSTP1 Ile105Val; a relative quantification method, to identify the copy number variation in GSTM1; and polymerase chain reaction followed by gel electrophoresis, to identify the null vs. non-null genotypes of GSTM1. The association between genotypes and OS of patients was assessed using Kaplan-Meier survival curves and Cox proportional hazards regression analysis. The OS of patients treated with paclitaxel + carboplatin-based chemotherapy was significantly increased, compared with patients treated with alternative forms of chemotherapy $(\mathrm{P}=0.035)$. The $\mathrm{OS}$ of patients did not differ significantly between different GSTP1 genotypes (log-rank test, $\mathrm{P}=0.17$ ). Cox proportional hazards regression analysis revealed that, since the start of the treatment, there was not a significant association between the GSTP1 isoleucine allele and the OS for heterozygous carriers of the isoleucine allele [hazards ratio (HR), 1.78; 95\% confidence interval $(\mathrm{CI}), 0.77-4.12 ; \mathrm{P}=0.18]$ and no homozygous carriers of the valine allele had been detected (HR, 0.00). There was no significant difference between GSTM1 genotypes, according to
\end{abstract}

Correspondence to: Dr An-Cong Wang, Department of Reproductive Medicine, Linyi People's Hospital, 27 Jiefang Road, Linyi, Shandong 276003, P.R. China

E-mail: ancongw12@163.com

Key words: single nucleotide polymorphisms, chemotherapy, pyrosequencing, ovarian serous cystadenocarcinoma, GSTM1, GSTP1, carboplatin, pharmacogenetics
Kaplan-Meier survival analysis (log-rank test, $\mathrm{P}=0.83$ ). Patients that possessed $\leq 1$ copy of GSTM1 exhibited no decrease in OS (HR, 0.96; 95\% CI, 0.37-2.51; $\mathrm{P}=0.94$ ), compared with patients that possessed two copies of GSTM1 (HR, 0.71; 95\% $\mathrm{CI}, 0.22-2.28 ; \mathrm{P}=0.56)$. Overall, the present results suggest that there are no associations between polymorphisms in the GSTP1 and GSTM1 genes and the OS of patients with ovarian cancer following administration of adjuvant chemotherapy.

\section{Introduction}

Ovarian cancer is the sixth most common type of cancer diagnosed in women worldwide, accounting for $\sim 4 \%$ of all types of female cancer, and is the second most common gynecological cancer, with $\sim 75 \%$ of patients presenting with late-stage disease (1-3). Patients with ovarian cancer are generally treated with first-line chemotherapy, including paclitaxel and platinum-based drugs (4). Cisplatin, the first-generation platinum-based drug, was gradually replaced by carboplatin and oxaliplatin, which are second- and third-generation platinum-based drugs, respectively $(5,6)$. Cisplatin was replaced due to liver and kidney toxicity, in addition to side effects such as mucositis, neutropenia and alopecia. Since the genetic profiles of patients with cancer have been associated with drug metabolism, patients may receive targeted chemotherapy, which avoids unnecessary toxicity (7). Pharmacogenetics is important in cancer chemotherapy, and the prognosis of patients with cancer may be explained according to their genetic background (8). The effects of platinum-based drugs are controlled by genes involved in detoxification, including glutathione S-transferase (GST)P1 and GSTM1 (9).

GST belongs to the multifunctional poly-2 protein family, and is primarily responsible for catalyzing the interaction between reduced glutathione hormone and electrophilic substances (10). GST is important in protecting tissues from damage caused by oxidative stress (11). The GSTM1 gene has two variants, and encodes an enzyme that participates in the metabolism of carcinogens (12).

The influence of genetic polymorphisms in the different response to platinum-based chemotherapy has been observed in certain respiratory and digestive types of cancer, including 
non-small cell lung and colorectal cancer (13-17). However, to the best of our knowledge, genetic polymorphisms in patients with ovarian cancer who have been treated with paclitaxel and platinum-based therapies have not been characterized thus far. Therefore, the present study aimed to investigate the association between genetic polymorphisms in drug metabolism and the overall survival (OS) of patients with ovarian cancer treated with paclitaxel and carboplatin-based chemotherapy. The present study evaluated specific polymorphic genes, namely GSTP1 Ile105Val and GSTM1 null, in order to assess the association between the phenotype pattern and the OS of chemotherapy-treated patients with ovarian serous cystadenocarcinoma.

\section{Materials and methods}

Patients and tissue samples. Tissue samples from 95 patients with ovarian serous cystadenocarcinoma (median age, 53.6 \pm 11.5 years) were obtained from Linyi People's Hospital (Linyi, China). All the patients were diagnosed and treated at the Linyi People's Hospital between August 2005 and July 2013. The tissue samples were obtained during diagnostic or therapeutic surgery. The patients were followed-up once every 2-4 months for 2 years and then every 3-6 months in the subsequent 3 years. Patients required follow-up once a year from 5 years onwards. The median follow-up of patients that were alive at the end of the present study was $25.6 \pm 14.3$ months (range, 3.0-92.0 months) All the patients provided written informed consent for the use of tissues samples and participation in the study. The present study was approved by the ethics committee at Linyi People's Hospital.

Data from 104 patients was collected for the present study, of which 9 patients were excluded. Patients that lacked information regarding cancer stage or start dates of chemotherapeutic treatment were excluded from the analysis. In addition, patients with a survival time of $<1$ month were excluded from the study. Information regarding the chemotherapeutic treatment regimen was available for all the patients included in the study. If the patients that possessed stage II-IV cancer, who were treated with 2 cycles of paclitaxel and carboplatin-based neoadjuvant chemotherapy (TP regimen) and 4-6 cycles of chemotherapy post-surgery, were allergic to paclitaxel, their levels of cancer antigen 125 were elevated following 1-2 chemotherapy cycles or computed tomography revealed that the volume of the tumor was not reduced, then the patients would be administered a different chemotherapy regimen, namely gemcitabine $\left(1,000 \mathrm{mg} / \mathrm{m}^{2}\right.$, on days 1 and 8 ; treatment cycle, $21-28$ days), ifosfamide $\left(1,700 \mathrm{mg} / \mathrm{m}^{2}\right.$, on days $1-3$; treatment cycle, $21-28$ days) or topotecan $\left(0.75 \mathrm{mg} / \mathrm{m}^{2}\right.$, on days $1-5$; treatment cycle, 21-28 days). In total, 95 patients that received adjuvant or palliative chemotherapy, of which 56 patients received TP regimen and 39 patients received alternative chemotherapy, were eligible for analysis. The patients that received the TP regimen were intravenously administered with $135-175 \mathrm{mg} / \mathrm{m}^{2}$ paclitaxel and $300-400 \mathrm{mg} / \mathrm{m}^{2}$ carboplatin on days 1 and 2, respectively. The treatment cycle was 21-28 days. The characteristics of the patients are presented in Table I.

Genotyping of GST. The tumor tissues were obtained from Department of Pathology, Linyi People's Hospital, where the pathologists used the following procedure: Following the
Table I. Characteristics of 95 patients with ovarian serous cystadenocarcinoma.

\begin{tabular}{lc} 
Characteristic & Value \\
\hline Age, years & \\
Median & 53.6 \\
Range & $20.0-80.0$ \\
Follow-up time, months & \\
Median & 25.6 \\
Range & $3.0-92.0$ \\
Chemotherapy, n (\%) & \\
Paclitaxel + carboplatin & $56(58.9)$ \\
Other & $39(41.1)$ \\
Tumor stage, n (\%) & \\
II & $11(11.6)$ \\
III & $63(66.3)$ \\
IV & $21(22.1)$ \\
\hline
\end{tabular}

surgery, the tumor specimens were immediately fixed with $10 \%$ formalin (Xilong Chemical Co., Ltd., Shantou, China) for $24 \mathrm{~h}$. Subsequent to rinsing with running tap water, the tissues were dehydrated with 70,80 and 95\% ethanol, followed by $100 \%$ ethanol, which was changed 3 times. Subsequent to clearing with xylene (twice), the tissues were immersed in paraffin 3 times (Leica, Wetzlar, Germany). The paraffin-embedded tissue blocks were cut into $10-\mu \mathrm{m}$ thick sections, from which genomic DNA was extracted using the Wizard ${ }^{\circledR}$ Genomic DNA Purification Kit (Promega Corporation, Madison, WI, USA). The germline mutations of GSTP1 and GSTM1 were analyzed in all patients using pyrosequencing. A total of four variants [three single nucleotide polymorphisms (SNPs) and one gene deletion] were assessed using polymerase chain reaction (PCR) and pyrosequencing, as previously described (18).

The specific sequence primers (Shanghai Shengong Biotechnology, Shanghai, China) used were as follows: GSTM1, forward, 5'-CGCCATCTTGTGCTACATTGC-3'; reverse, 5'-CACAAATTCTGGATTGTAGCAGA-3'; and P (the reverse primer resulting in a 237 bp fragment), 5'-GGC CTCCTCCTTGGCTGG-3'; GSTP1 Ile105Val, forward, 5'-AATGACGGCGTGGAGGAC-3'; reverse, 5'-GGTCAG CCCAAGCCACCT-3'; and $\mathrm{P}$ (the reverse primer resulting in a 155 bp fragment), 5'-AGGACCTCCGCTGCAAAT-3'. The PCR cycle conditions were as follows: Denaturation at $95^{\circ} \mathrm{C}$ for $5 \mathrm{~min}$, followed by 35 cycles of $95^{\circ} \mathrm{C}$ for $45 \mathrm{sec}, 60^{\circ} \mathrm{C}$ for $30 \mathrm{sec}$ and $72^{\circ} \mathrm{C}$ for $30 \mathrm{sec}$. DNA amplification was performed using Taq DNA Polymerase Master Mix RED (Biomol GmbH, Hamburg, Germany). For quality control purposes and to verify the results, $10 \%$ of samples were re-analyzed and $100 \%$ concordance was indicated (19). Negative control samples were included in each amplification series. The presence of one or both GSTM1 alleles, as identified by the presence of a 237 bp fragment or complete deletion (null genotype), was analyzed by electrophoresis on a $1.2 \%$ agarose gel using DL500 as a DNA marker (Takara Bio, Dalian, China). 
Table II. HR of the overall survival of 95 patients with ovarian serous cystadenocarcinoma, according to their GSTP1 and GSTM1 polymorphisms.

\begin{tabular}{|c|c|c|c|c|c|c|}
\hline Genetic polymorphism & Patients, $\mathrm{n}$ & Mortalities, $\mathrm{n}$ & P-value ${ }^{a}$ & HR & $95 \% \mathrm{CI}$ & P-value ${ }^{b}$ \\
\hline \multicolumn{7}{|l|}{ GSTP1 } \\
\hline $\mathrm{A} / \mathrm{A}$ & 37 & 11 & - & 1.00 & - & - \\
\hline $\mathrm{A} / \mathrm{G}$ & 58 & 11 & 0.17 & 1.78 & $0.77-4.12$ & 0.18 \\
\hline $\mathrm{G} / \mathrm{G}$ & 0 & 0 & 0.00 & 0.00 & $0.00-0.00$ & 0.00 \\
\hline \multicolumn{7}{|l|}{ GSTM1 } \\
\hline Homozygous ( 2 copies) & 27 & 8 & - & 1.00 & - & - \\
\hline Heterozygous (1 copy) & 20 & 4 & - & 0.96 & $0.37-2.51$ & 0.94 \\
\hline Homozygous ( 0 copies $)$ & 48 & 10 & 0.83 & 0.71 & $0.22-2.28$ & 0.56 \\
\hline
\end{tabular}

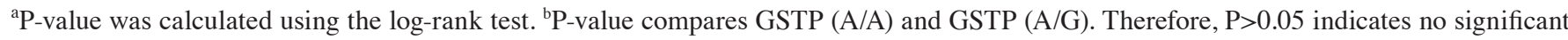
difference in the Cox proportional hazards regression analysis. HR=1.00 indicates the internal reference. Missing data are indicated by HR, hazards ratio; GST, glutathione S-transferase; CI, confidence interval.

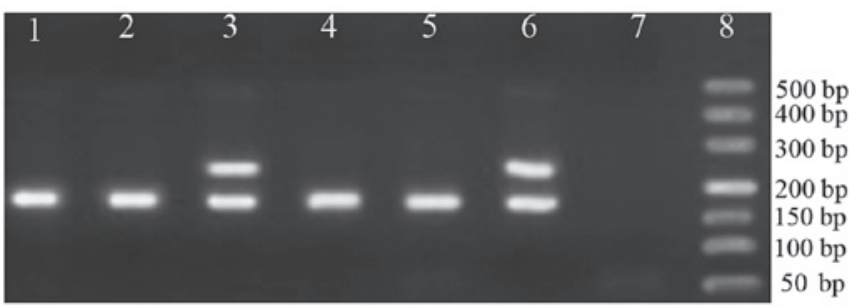

Figure 1. Patient genotyping using multiplex-polymerase chain reaction Lanes 1, 2, 4 and 5 show samples from patients exhibiting the GSTM4 gene (null genotype), whereas lanes 3 and 6 contain samples obtained from patients exhibiting the GSTM1 and GSTM4 genes (non-null genotype). Lane 7, negative control. Lane 8, DL500 DNA Marker. GST, glutathione S-transferase.

Electrophoresis was performed for $30 \mathrm{~min}$ at $120 \mathrm{~V}$. The gel image was captured using BDAdigital (Analytik Jena AG, Jena, Germany). The absence of amplifiable GSTM1 (in the presence of the GSTM4 co-amplified control) indicated a null genotype.

GSTM1 gene expression analysis by reverse transcription-quantitative $P C R \quad(R T-q P C R)$. Relative complementary DNA quantitation for GSTM1 and an internal reference gene ( $\beta$-actin) was performed on formalin-fixed, paraffin-embedded surgical specimens from 95 patients. Following standard tissue sample deparaffinization using xylene and ethanol (20), samples were lysed in a tris-chloride, ethylenediaminetetraacetic acid, sodium dodecyl sulphate and proteinase K-containing buffer. RNA was then extracted with phenol-chloroform-isoamyl alcohol followed by precipitation with isopropanol in the presence of glycogen and sodium acetate. RNA was resuspended in diethyl pyrocarbonate water (Ambion, Inc.; Thermo Fisher Scientific) and treated with DNAseI (Ambion, Inc.; Thermo Fisher Scientific) to avoid DNA contamination. Complementary DNA was synthesized using the Moloney Murine Leukemia Virus retrotranscriptase enzyme. Template cDNA was added to Taqman Universal MasterMix (Applied Biosystems; Thermo Fisher Scientific, Inc.) in a $12.5-\mu 1$ reaction with specific primers and probes for each gene. The primer and probe sets were designed using PrimerExpress 2.0 Software (Applied Biosystems; Thermo Fisher Scientific, Inc.) and the RefSeq sequences (http://www.ncbi.nlm.nih.gov/entrez/query. fcgi? $\mathrm{db}=$ gene). The sequences of the primers and probes used were as follows: GSTM1 forward, 5'-CCCAGAGCA ACGCCATCT-3'; reverse, 5'-TCCACACGAATCTTCTCC TCTTC-3'; probe, (FAM)-5'-CTACATTGCCCGCAAGCA CAACCTG-3'-(TAMRA). $\beta$-actin (internal reference gene) forward, 5'-TGAGCGCGGCTACAGCTT-3'; reverse, 5'-TCC TTAATGTCACGCACGATTT-3'; probe, (FAM)-5'-ACC ACCACGGCCGAGCGG-3'-(TAMRA). Quantification of gene expression was carried out using the LineGene $\mathrm{K}$ system (Bioer Technology Co., Ltd.).

Relative gene expression quantification was calculated according to the comparative cycle threshold $(\mathrm{Cq})$ method using $\beta$-actin as an endogenous control Final results were determined as follows: $2^{-\Delta \Delta \mathrm{Cq}}$, where $\Delta \mathrm{Cq}$ values of the sample are determined by subtracting the $\mathrm{Cq}$ value of the target gene from the value of the $\beta$-actin gene.

Statistical analysis. SPSS software version 17.0 (SPSS Inc., Chicago, IL, USA) was used for statistical analysis. $\mathrm{P}<0.05$ was considered to indicate a statistically significant difference. All statistical tests were two-sided with a significance level of $\alpha=0.05$. Genotype data were categorized as follows: Homozygous carriers of the wild-type allele for GSTP1 polymorphism (Val105 Val); carriers of the heterozygous allele or possessing one gene copy (GSTP1 Ile105Val); and carriers of the homozygous variant allele or possessing no copy (GSTP1 Ile105Ile). Patients with >1 copy of GSTM1 were considered to exhibit the non-null genotype, whereas patients possessing no copies of GSTM1 were considered to exhibit the null genotype. The OS of patients was calculated as the time between the start of the chemotherapeutic treatment and the date of the last follow-up or the date when the patient succumbed to disease. Kaplan-Meier survival function analysis was used to evaluate the association between GST genotypes and the OS of patients. The log-rank test was used to calculate the difference between OS and genotype. Cox proportional hazards regression analysis was used to calculate 

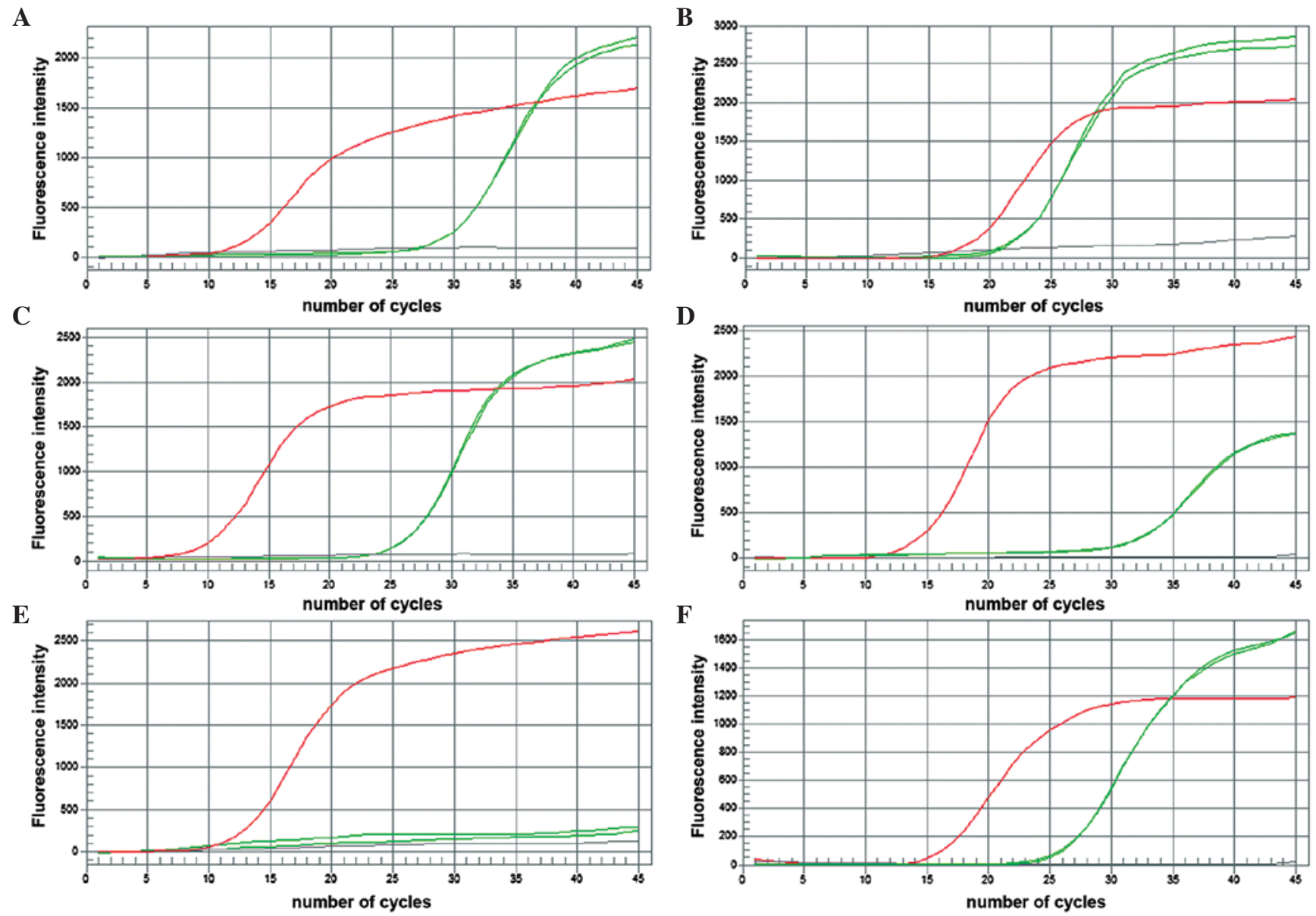

Figure 2. Amplification curves obtained during multiplex-polymerase chain reaction. (A) Sample containing one copy of the GSTM1 gene. The red line represents the positive reference amplification curve for GSTM1, the green line represents the amplification curve for GSTM1 and the black line represents the amplification curve for the negative reference for GSTM1. (B) Amplication curve for the internal reference gene, $\beta$-actin for the sample containing one copy of the GSTM1 gene. The red line represents the amplification curve for the positive reference for $\beta$-actin, the green line represents the amplification curve for $\beta$-actin and the black line represents the amplification curve for the negative reference for $\beta$-actin. (C) Amplification curves for samples containing two copies of the GSTM1 gene. (D) Amplication curve for the internal reference gene, $\beta$-actin, for the sample containing two copies of the GSTM1 gene. (E) Amplification curves for samples exhibiting the null phenotype. (F) Amplication curve for the internal reference gene, $\beta$-actin, for the sample exhibiting the null phenotype. GST, glutathione S-transferase.

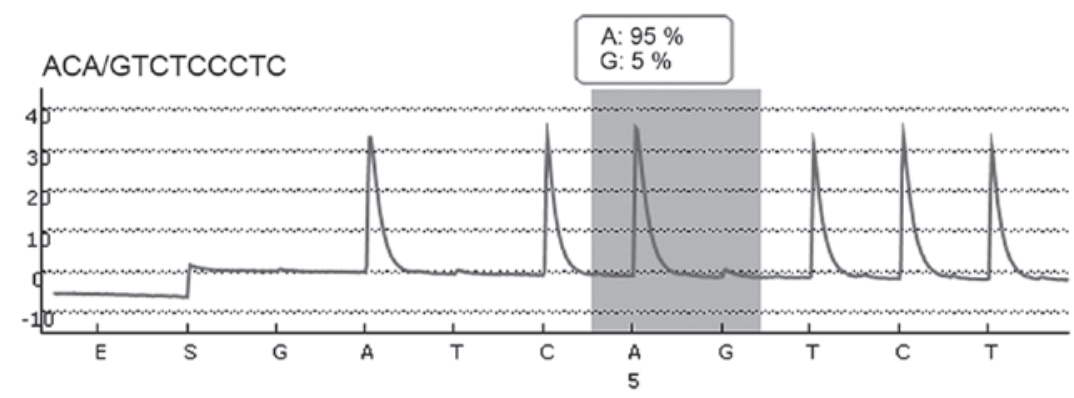

Figure 3. Genotype sequence of homozygous glutathione S-transferase P1 Ile105Val, ACA/GTCT (A/A). E, enzyme mixture (DNA polymerase, ATP sulfurylase, luciferase, apyrase); S, substrate (5-phosphoryl sulfate, fluorescein).

multivariable adjusted hazard ratios (HRs) and their corresponding $95 \%$ confidence interval (CI).

\section{Results}

The frequency of GSTM1 positive and negative individuals was determined by genotyping using multiplex-PCR as shown in Fig. 1. Relative GSTM1 gene expression was also quantified (Fig. 2).

OS of patients with ovarian serous cystadenocarcinoma. Patients that received TP or an alternative form of chemotherapy were followed-up for a median time of $25.6 \pm 14.3$ months. Kaplan-Meier survival analysis demonstrated that the OS of 


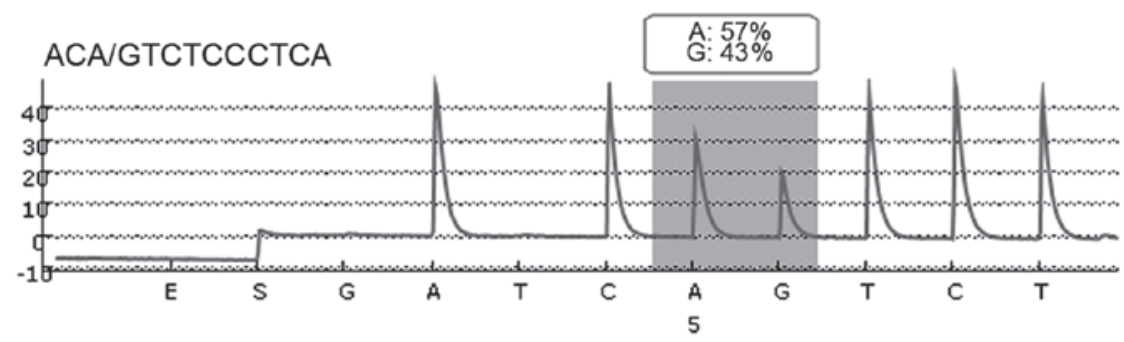

Figure 4. Genotype sequence of heterozygous carriers of glutathione S-transferase P1 Ile105Val, ACA/GTCT (A/G). E, enzyme mixture (DNA polymerase, ATP sulfurylase, luciferase, apyrase); S, substrate (5-phosphoryl sulfate, fluorescein).

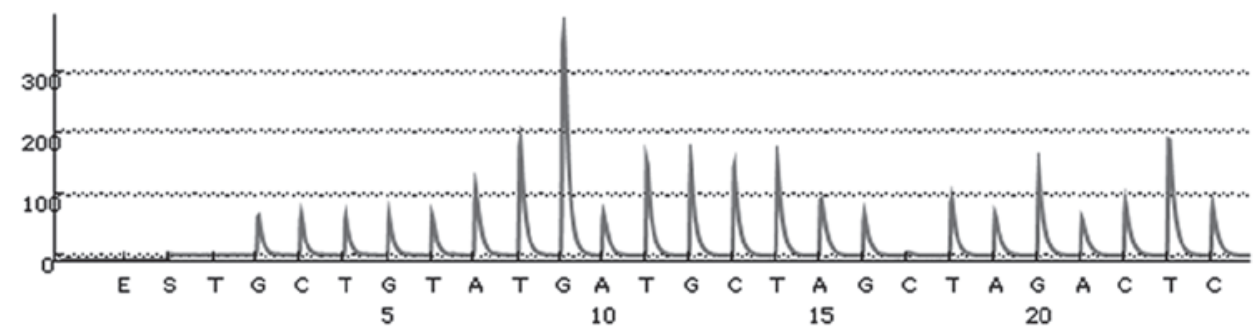

Figure 5. Genotype sequence of glutathione S-transferase M1 non-null, TTGGCCTTAGC/TAGGACTTTC. E, enzyme mixture (DNA polymerase, ATP sulfurylase, luciferase, apyrase); S, substrate (5-phosphoryl sulfate, fluorescein).

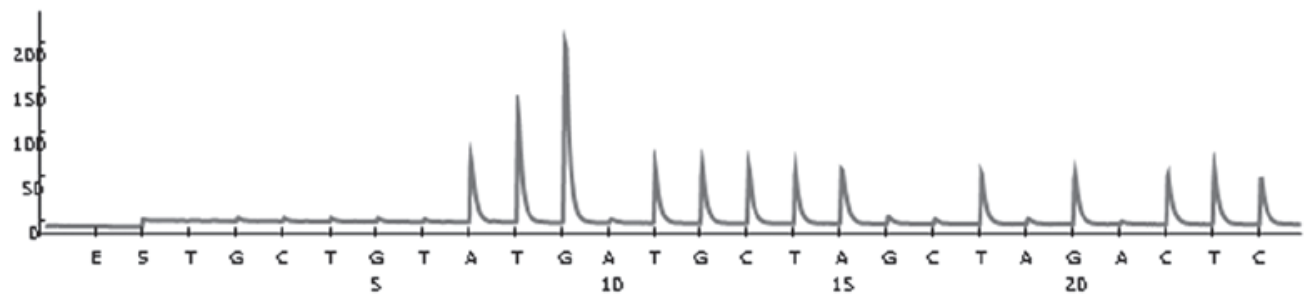

Figure 6. Genotype sequence of glutathione S-transferase M1 null, ATTGGGGTGCTATGCTC. E, enzyme mixture (DNA polymerase, ATP sulfurylase, luciferase, apyrase); $\mathrm{S}$, substrate (5-phosphoryl sulfate, fluorescein).

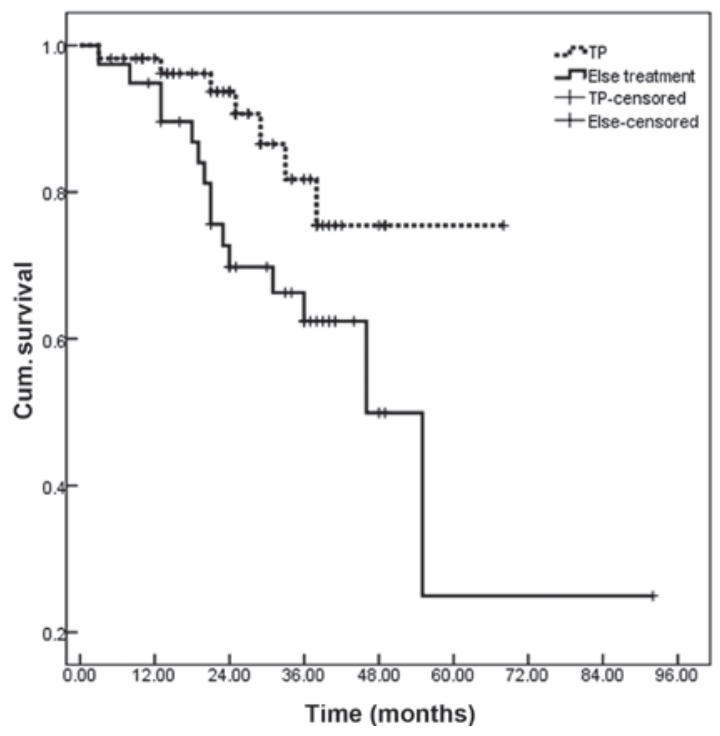

Figure 7. Overall survival of patients with ovarian serous cystadenocarcinoma that were administered with paclitaxel + carboplatin-based chemotherapy or an alternative chemotherapeutic drug (long-rank test, $\mathrm{P}=0.035$ ). TP, paclitaxel + carboplatin-based chemotherapy; Else, non-TP chemotherapy; Cum., cumulative. patients did not differ significantly between GSTP1 genotypes (log-rank test, $\mathrm{P}=0.17$ ). Cox proportional hazards regression analysis revealed that, since the start of treatment, there was not a significant association between the GSTP1 isoleucine allele and OS for heterozygous carriers of the isoleucine allele (HR, 1.78; 95\% CI, 0.77-4.12; $\mathrm{P}=0.18$ ) and no homozygous carriers of the valine allele had been detected (HR, 0.00).

Kaplan-Meier survival analysis also revealed that the OS did not differ significantly between GSTM1 genotypes (log-rank test, $\mathrm{P}=0.83$ ). Compared with carriers of two copies of GSTM1, patients with $\leq 1$ copies of GSTM1 exhibited no decrease in the risk of mortality following chemotherapy [HR, $0.96,95 \% \mathrm{CI}, 0.37-2.51(\mathrm{P}=0.94)$ for patients with one copy of GSTM1 vs. HR, 0.71, 95\% CI, 0.22-2.28 ( $\mathrm{P}=0.56)$ for patients with no copies of GSTM1, respectively] (Table II; Figs. 3-6).

Overall, there were no associations between polymorphisms in GSTP1 and GSTM1 and the OS of patients following adjuvant chemotherapy. However, the OS of patients administered with TP was significantly increased, compared with patients that received other type of chemotherapy ( $\mathrm{P}=0.035$; Fig. 7). 


\section{Discussion}

Platinum-based chemotherapy drugs are commonly used to treat certain solid tumors, including non-small cell lung and colorectal cancer, in order to induce the formation of DNA adducts, which contributes to the death of tumor cells (21). However, DNA repair and drug metabolism may hinder the prognosis of patients that are administered platinum-based chemotherapy $(22,23)$.

Variations in the GSTP1 and GSTM1 genes have been extensively studied, due to their capacity to modulate the drug response in patients with cancer (24). GSTM1 deficiency has been reported to increase the risk of developing head-neck tumors, squamous cell carcinoma, and lung, colorectal, bladder and breast cancer (25-27).

The present study aimed to evaluate the association between genetic polymorphisms in the GSTP1 and GSTM1 genes and the OS of patients with ovarian serous cystadenocarcinoma treated with chemotherapy. GSTP1 is a member of the GST superfamily, and is important in the defense function of cells (28). GSTP1 Ile105Val polymorphism leads to a decreased ability in cell defense, thus increasing the sensitivity of an individual to platinum-based chemotherapy $(29,30)$. However, the results of the Cox proportional hazards regression in the present study revealed no significant association between the GSTP1 Ile105Val isoleucine allele, and no homozygous carriers of the valine allele were detected. None of the polymorphisms in the GSTP1 and GSTM1 genes that were evaluated in the present study were observed to be significantly associated with the OS of patients that received chemotherapy TP regimen or an alternative chemotherapeutic drug. Similarly, a previous study conducted on 65 patients with colorectal cancer that received first-line chemotherapy of oxaliplatin did not identify an association between GSTP1 genotype and survival (16). However, a dose-dependent association between the number of GSTP1 valine alleles and survival in certain patients with cancer that received second-line oxaliplatin treatment was reported by a previous study, although no association between GSTM1 genotype and survival was observed (31). The present results revealed that there was not a significant association between the GSTP1 isoleucine allele and the risk of mortality for heterozygous carriers of the isoleucine allele (HR, 1.78; 95\% CI, 0.77-4.12; $\mathrm{P}=0.18$ ) and homozygous carriers of the valine allele (HR, 0.00 ; no homozygous carriers of the valine allele were detected) using Cox proportional hazards regression analysis.

Townsend and Tew (32) reported that GSTM1 is important in the detoxification of various carcinogens, and is associated with the metabolism of various chemotherapeutic agents. The results of the present study revealed that the OS of patients did not vary significantly according to their GSTM1 genotype. Patients with $\leq 1$ copies of GSTM1 exhibited no decreased risk of mortality following chemotherapy, compared with patients with two copies of GSTM1.

In conclusion, the present study demonstrates that there is a decreased risk of mortality for chemotherapy-treated patients that have a reduced copy number of the GSTM1 allele. In addition, patients that were GSTP1 heterozygous carriers of the valine allele $(\mathrm{A} / \mathrm{G})$ had an increased risk of mortality, which is contrary to the results of other studies $(33,34)$.
Tumor characteristics, including classification and stage, may have resulted in the inconsistent results observed. Additional studies with larger sample sizes are required to confirm the results of the present study.

\section{Acknowledgements}

The present study was supported by the Medical and Health Science Technology Development Project of Shandong Province, China (grant no. 2015WS0377) and the Natural Science Foundation of Shandong Province, China (grant no. ZR2010CQ035).

\section{References}

1. Jemal A, Siegel R, Ward E, Murray T, Xu J and Thun MJ: Cancer statistics, 2007. CA Cancer J Clin 57: 43-66, 2007.

2. Parkin DM, Bray F, Ferlay J and Pisani P: Global cancer statistics, 2002. CA Cancer J Clin 55: 74-108, 2005.

3. Partridge EE and Barnes MN: Epithelial ovarian cancer: Prevention, diagnosis, and treatment. CA Cancer J Clin 49: 297-320, 1999.

4. Harries M and Gore M: Part I: Chemotherapy for epithelial ovarian cancer-treatment at first diagnosis. Lancet Oncol 3: 529-536, 2002.

5. Petrelli F, Zaniboni A, Coinu A, Cabiddu M, Ghilardi M, Sgroi G and Barni S: Cisplatin or not in advanced gastric cancer: A systematic review and meta-analysis. PLoS One 8: e83022, 2013.

6. Tan S, Peng X, Peng W, Zhao Y and Wei Y: Enhancement of oxaliplatin-induced cell apoptosis and tumor suppression by 3-methyladenine in colon cancer. Oncol Lett 9: 2056-2062, 2015.

7. Kweekel DM, Gelderblom H and Guchelaar HJ: Pharmacology of oxaliplatin and the use of pharmacogenomics to individualize therapy. Cancer Treat Rev 31: 90-105, 2005.

8. Jun L, Haiping Z and Beibei Y: Genetic polymorphisms of GSTP1 related to response to 5-FU-oxaliplatin-based chemotherapy and clinical outcome in advanced colorectal cancer patients. Swiss Med Wkly 139: 724-728, 2009.

9. Shim HJ, Yun JY, Hwang JE, Bae WK, Cho SH, Lee JH, Kim HN, Shin MH, Kweon SS, Lee JH, et al: BRCA1 and XRCC1 polymorphisms associated with survival in advanced gastric cancer treated with taxane and cisplatin. Cancer Sci 101: 1247-1254, 2010.

10. Yan H, Jia H, Gao H, Guo X and Xu B: Identification, genomic organization, and oxidative stress response of a sigma class glutathione S-transferase gene (AccGSTS1) in the honey bee, Apis cerana cerana. Cell Stress Chaperones 18: 415-426, 2013.

11. Vontas JG, Small GJ and Hemingway J: Glutathione S-transferases as antioxidant defence agents confer pyrethroid resistance in Nilaparvata lugens. Biochem J 357: 65-72, 2001.

12. Hayes JD and Pulford DJ: The glutathione S-transferase supergene family: Regulation of GST and the contribution of the isoenzymes to cancer chemoprotection and drug resistance. Crit Rev Biochem Mol Biol 30: 445-600, 1995.

13. Chan EC, Lam SY, Fu KH and Kwong YL: Polymorphisms of the GSTM1, GSTP1, MPO, XRCC1 and NQO1 genes in Chinese patients with non-small cell lung cancers: Relationship with aberrant promoter methylation of the CDKN2A and RARB genes. Cancer Genet Cytogenet 162: 10-20, 2005.

14. Ye F, Liu Z, Tan A, Liao M, Mo Z and Yang X: XRCC1 and GSTP1 polymorphisms and prognosis of oxaliplatin-based chemotherapy in colorectal cancer: A meta-analysis. Cancer Chemother Pharmacol 71: 733-740, 2013.

15. Economopoulos KP and Sergentanis TN: GSTM1, GSTT1, GSTP1, GSTA1 and colorectal cancer risk: A comprehensive meta-analysis. Eur J Cancer 46: 1617-1631, 2010.

16. Funke S, Timofeeva M, Risch A, Hoffmeister M, Stegmaier C, Seiler CM, Brenner H and Chang-Claude J: Genetic polymorphisms in GST genes and survival of colorectal cancer patients treated with chemotherapy. Pharmacogenomics 11: 33-41, 2010.

17. Zaanan A, Dalban C, Emile JF, Blons H, Fléjou JF, Goumard C, Istanbullu M, Calmel C, Alhazmi K, Validire P, et al: ERCC1, XRCC1 and GSTP1 single nucleotide polymorphisms and survival of patients with colon cancer receiving oxaliplatin-based adjuvant chemotherapy. J Cancer 5: 425-432, 2014. 
18. Marsh S, King CR, Garsa AA and McLeod HL: Pyrosequencing of clinically relevant polymorphisms. Methods Mol Biol 311: 97-114, 2005.

19. Butkiewicz D, Rusin M, Sikora B, Lach A and Chorąży M: An association between DNA repair gene polymorphisms and survival in patients with resected non-small cell lung cancer. Mol Biol Rep 38: 5231-5241, 2011.

20. Kizys MM, Cardoso MG,Lindsey SC, Harada MY, Soares FA, Melo MC, Montoya MZ, Kasamatsu TS, Kunii IS, Giannocco G, et al: Optimizing nucleic acid extraction from thyroid fine-needle aspiration cells in stained slides, formalin-fixed/paraffin-embedded tissues, and long-term stored blood samples. Arq Bras Endocrinol Metabol 56: 618-626, 2012.

21. Chai H, Pan J, Zhang X, Zhang X, Shen X, Li H, Zhang K, Yang C, Sheng $\mathrm{H}$ and Gao H: ERCC1 C118T associates with response to FOLFOX4 chemotherapy in colorectal cancer patients in Han Chinese. Int J Clin Exp Med 5: 186-194, 2012.

22. Chen YC, Tzeng CH, Chen PM, Lin JK, Lin TC, Chen WS, Jiang JK, Wang HS and Wang WS: Influence of GSTP1 I105V polymorphism on cumulative neuropathy and outcome of FOLFOX-4 treatment in Asian patients with colorectal carcinoma. Cancer Sci 101: 530-535, 2010.

23. Lv H, Li Q, Qiu W, Xiang J, Wei H, Liang H, Sui A and Liang J: Genetic polymorphism of XRCC1 correlated with response to oxaliplatin-based chemotherapy in advanced colorectal cancer. Pathol Oncol Res 18: 1009-1014, 2012.

24. Ali-Osman F, Akande O, Antoun G, Mao JX and Buolamwini J: Molecular cloning, characterization and expression in Escherichia coli of full-length cDNAs of three human glutathione $\mathrm{S}$-transferase Pi gene variants. Evidence for differential catalytic activity of the encoded proteins. J Biol Chem 272: 10004-10012, 1997.

25. Godschalk RW, Dallinga JW, Wikman H, Risch A, Kleinjans JC, Bartsch H and Van Schooten FJ: Modulation of DNA and protein adducts in smokers by genetic polymorphisms in GSTM1, GSTT1, NAT1 and NAT2. Pharmacogenetics 11: 389-398, 2001.

26. Gertig DM, Stampfer M, Haiman C, Hennekens CH, Kelsey K and Hunter DJ: Glutathione S-transferase GSTM1 and GSTT1 polymorphisms and colorectal cancer risk: A prospective study. Cancer Epidemiol Biomarkers Prev 7: 1001-1005, 1998

27. Slattery ML, Potter JD, Samowitz W, Bigler J, Caan B and Leppert M: NAT2, GSTM-1, cigarette smoking, and risk of colon cancer. Cancer Epidemiol Biomarkers Prev 7: 1079-1084, 1998.
28. Li QF, Yao RY, Liu KW, Lv HY, Jiang T and Liang J: Genetic polymorphism of GSTP1: Prediction of clinical outcome to oxaliplatin/5-FU-based chemotherapy in advanced gastric cancer. J Korean Med Sci 25: 846-852, 2010.

29. Hong J, Han SW, Ham HS, Kim TY, Choi IS, Kim BS, Oh DY, Im SA, Kang GH, Bang YJ and Kim TY: Phase II study of biweekly S-1 and oxaliplatin combination chemotherapy in metastatic colorectal cancer and pharmacogenetic analysis. Cancer Chemother Pharmacol 67: 1323-1331, 2011.

30. Watson MA, Stewart RK, Smith GB, Massey TE and Bell DA: Human glutathione S-transferase P1 polymorphisms: Relationship to lung tissue enzyme activity and population frequency distribution. Carcinogenesis 19: 275-280, 1998 .

31. Stoehlmacher J, Park DJ, Zhang W, Groshen S, Tsao-Wei DD, $\mathrm{Yu} \mathrm{MC}$ and Lenz HJ: Association between glutathione S-transferase P1, T1, and M1 genetic polymorphism and survival of patients with metastatic colorectal cancer. J Natl Cancer Inst 94: 936-942, 2002.

32. Townsend DM and Tew KD: The role of glutathione-S-transferase in anti-cancer drug resistance. Oncogene 22: 7369-7375, 2003.

33. Huang MY, Huang ML, Chen MJ, Lu CY, Chen CF, Tsai PC, Chuang SC, Hou MF, Lin SR and Wang JY: Multiple genetic polymorphisms in the prediction of clinical outcome of metastatic colorectal cancer patients treated with first-line FOLFOX-4 chemotherapy. Pharmacogenet Genomics 21: 18-25, 2011.

34. Suh KW, Kim JH, Kim DY, Kim YB, Lee C and Choi S: Which gene is a dominant predictor of response during FOLFOX chemotherapy for the treatment of metastatic colorectal cancer, the MTHFR or XRCC1 gene? Ann Surg Oncol 13: 1379-1385, 2006. 